\title{
PROKLA-Redaktion
}

\section{Editorial: Gesundheit mit System}

D er Beginn der Covid-19-Pandemie liegt inzwischen fast zwei Jahre zurück. In Anbetracht von knappen medizinischen Behandlungs- und Bettenkapazitäten sowie Liefer- und Produktionsengpässen diverser Art, ist die Notwendigkeit einer funktionierenden Notfall-, Gesundheits- und Arzneiversorgung schlagartig in den Fokus von Politik, Wissenschaft und Öffentlichkeit gerückt - und damit auch die Bedingungen ihres Gelingens. In den letzten Monaten wurde herausgestellt, dass die Pandemie wie ein Brennglas wirke. Covid-19 sei ein »Stresstest für Gesundheitssysteme«. Es wurde auch deutlich, dass die zunehmende Ausrichtung der Gesundheitspolitik der letzten 30 Jahre an wettbewerbsstaatlichen Prinzipien nicht spurlos an der Gesundheitsversorgung vorbei gingen: Das politische Anliegen einer Verbesserung der gesundheitlichen Lage der Bevölkerung konkurriert mit der Strategie der Kostendämpfungspolitik, in der Gesundheitsrisiken und -kosten zunehmend privatisiert werden.

Offensichtlich fehlen Investitionen in das Gesundheitssystem, Einsparungen sind nicht zuletzt häufig Folgen einer Politik der Austerität. Deutlich zeigte sich dies in der Corona-Pandemie am Öffentlichen Gesundheitsdienst, der in der Pandemiebekämpfung zwar eine zentrale Rolle einnimmt, jedoch kaum seinen Aufgaben nachkommen kann. Mittel für Prävention und Gesundheitsförderung bleiben häufig aus, gerade diese aber könnten wichtige Impulse setzen, die gesundheitlichen Folgen sozialer Ungleichheit abzumildern. Denn Gesundheit ist auch eine Klassenfrage: Menschen, die an oder unter der Armutsgrenze leben, haben eine deutlich geringere Lebenserwartung als wohlhabende Menschen. Bei Männern beträgt dieser Unterschied im Schnitt 10,8 Jahre, bei Frauen 8 Jahre. Auch beim Zugang zu Gesundheitsleistungen selbst gibt es einen deutlichen Klassenbias, der nicht nur, wie häufig angenommen, Menschen ohne Krankenversicherungsschutz betrifft, sondern ganze Bevölkerungsgruppen.

Die am Solidaritätsprinzip ausgerichtete Gesetzliche Krankenversicherung (GKV), der etwa 90 Prozent der Versicherten angehören, sieht sich dem Profitmodell der Privaten Krankenversicherungen (PKV) gegenüber. Die medizinische Unterversorgung in bestimmten, vorwiegend ländlichen Regionen besteht oftmals entweder fort oder verschärft sich, wohingegen es in reicheren Gegenden weiterhin häufig zu einer Überversorgung mit Fach- und Allgemeinärzt*innen kommt. Privatwirtschaftliche Akteure stellen einen 
immer größeren Teil der Versorgungsstrukturen, ob nun Pflegeheime, ambulante Pflegedienste, Kliniken oder unlängst auch Medizinische Versorgungszentren.

Zuletzt haben wir uns in der PROKLA 4/2003 dem Schwerpunkt Gesundheit im Neoliberalismus gewidmet. Das Jahr stellte dabei eine Zäsur dar, da ab 2004 die bundesweite Anwendung der Fallpauschalen in den Krankenhäusern verpflichtend wurde. Diese ermöglichte es fortan, einzelnen Krankenhäusern, Gewinne und Verluste zu erwirtschaften und zwang sie damit in einen Wettbewerb. Die Folgen des daraus resultierenden Kostendrucks waren nicht selten schlechte Arbeitsbedingungen, eine geringere Vergütung und Personalmangel in der Krankenhauspflege und Outsourcing krankenhausbezogener Dienstleistungen. Die Behandlungsqualität für Patient*innen ist teilweise gesunken, Sorgeverantwortung wird nach der Entlassung weitergereicht und Angehörige müssen immer häufiger die Versäumnisse des Systems auffangen. Die Folgen haben inzwischen vermehrt Widerspruch hervorgerufen. Die im Jahr 2020 erfolgte Herauslösung der Pflegepersonalkosten aus den Fallpauschalen lässt sich als erster Schritt in Richtung eines möglichen Ausstiegs aus dem Fallpauschalensystem deuten, dem lange soziale Kämpfe vorausgingen. Bereits seit einigen Jahren gibt es eine gewerkschaftliche Initiative, häufig im Bündnis mit sozialen Bewegungen, die sich für Entlastung durch eine verbesserte Personalmindestbesetzung auf Stationen in Krankenhäusern engagiert. Die Arbeitskämpfe an den Krankenhäusern fanden im Herbst 2021 einen erneuten
Höhepunkt in Berlin, als Pflegekräfte, Hebammen und Reinigungskräfte der Vivantes und Charité gemeinsam in einen unbefristeten Streik traten - und zwar mit der Forderung nach einem gemeinsamen Tarifvertrag zur Verbesserung der Arbeitsbedingungen. An Auseinandersetzungen wie diesen wird auch deutlich, dass Gesundheitspolitik ein Feld gesellschaftlicher Machtkämpfe bildet, in dem der Zugang zu Gesundheitsleistungen zwischen öffentlichen und privatwirtschaftlichen Interessen ausgehandelt wird.

In diesem Heft wird das Thema Gesundheit auf mehreren Ebenen diskutiert. Der Beitrag von Michael Simon beschreibt die Entstehung sowie die zentralen Inhalte eines in den 1980erJahren entwickelten neoliberalen Reformmodells für den marktwirtschaftlichen Umbau des deutschen Gesundheitswesens und zeigt die Umsetzung des Reformmodells am Beispiel der Gesetzlichen Krankenversicherung (GKV). Ausgangspunkt war dabei das Gesundheitsstrukturgesetz von 1992, mit dem Wettbewerbselemente in das System der Krankenkassen eingeführt wurden. Die folgenden Reformen zielten allesamt darauf ab, eine staatliche Krankenversicherung durch einen Versicherungstarif für eine Basisabsicherung zu ersetzen, der sowohl von Krankenkassen als auch von privaten Krankenversicherungen angeboten wird. Während Michael Simon die Transformation der GKV in den Blick nimmt, beschäftigt sich Kalle Kunkel mit dem Fallpauschalensystem. Dabei sieht er zwei durchaus im Widerstreit befindliche Reformlogiken am Werk: zum einen die der Kostendämpfung, zum anderen 
die der Vermarktlichung. Für Kunkel ist die Einführung der Fallpauschalen weniger dem austeritätspolitischen Prinzip der Kostensenkung zuzuschreiben, sondern Folge der Logik der Vermarktlichung von Prozessen, die damit der politischen und demokratischen Steuerung entzogen werden sollen.

Thomas Gerlinger, Uwe Lenhard und Philipp Florian Schmid widmen sich in ihrem Artikel der Gesundheitsförderung und Krankheitsprävention und skizzieren dabei Entwicklungstendenzen und Widersprüche. Mit Blick auf die Arbeitswelt stellen sie fest, dass die Schwächung von gesetzlichen Interessenvertretungen häufig auch zulasten der gesundheitlichen Qualität von Arbeitsbedingungen geht. Zudem werden insbesondere die mit Formen der indirekten Steuerung wachsenden psychischen Belastungen zu selten Gegenstand von Maßnahmen eines wirksamen betrieblichen Arbeits- und Gesundheitsschutzes. Darüber hinaus widmen sie sich dem Aufstieg der gesetzlichen Krankenkassen zu wichtigen Akteuren der Gesundheitsförderung und Primärprävention, der erst in den letzten Jahren per Gesetz auf den Weg gebracht wurde.

Anna Weber beschäftigt sich in ihrem Beitrag mit dem pharmazeutischen Forschungs- und Produktionsregime. Dieses führte bereits vor der Pandemie zu einer mangelhaften Bedarfsdeckung, die sich in der Krise verschärft darin offenbart, dass der globale Zugang zu Impfstoffen gegen Corona ausgesprochen ungleich ist. Sie betont, dass Prozesse der Finanzialisierung ein bestehendes Missverhältnis zu Gunsten der Aufwertung von intellektuellen Eigen- tumsrechten verstärken und zulasten einer Verfügbarkeit und Erschwinglichkeit von dringend benötigten pharmazeutischen Produkten gehen.

Im kommenden Heft $(1 / 2022)$ werden wir uns explizit der Covid-19Pandemie zuwenden und einige im vorliegenden Heft aufgeworfene Fragen nochmals aufgreifen. Jenseits des Schwerpunkts setzt Margit Mayer ihren Artikel aus der PROKLA 2/2021 zu den Perspektiven der US-Linken nach Trump fort. Der zweite Teil des Aufsatzes präsentiert zunächst ein Update des Kontexts, in dem die neue BidenHarris-Regierung und die demokratische Mehrheit im Kongress agieren. Einige der zentralen Reformprojekte werden vorgestellt und bezüglich ihrer Implikationen für Bewegungshandeln bewertet. Es zeigt sich, dass Organisationen wie die Democratic Socialists of America (DSA) und Black Lives Matter (BLM) sich angesichts der aggressiven Politik der republikanischen Rechten und der fragilen Mehrheit für die Reformprojekte der Demokraten stärker als zuvor auf elektorale Politik orientieren und näher an die Demokratische Partei heranrücken.

Zwei weitere Texte diskutieren ein Thema, das bereits in früheren Heften Gegenstand war, die Frage nach Bedeutung von Gewalt und strukturellen Zwängen für bürgerliche Gesellschaften. Søren Mau argumentiert, dass den Kapitalismus eine besondere Form der Macht auszeichnet, den stummen Zwang der ökonomischen Verhältnisse, die sich weder auf Ideologie noch auf Gewalt reduzieren lasse und bisher kaum sozialwissenschaftlich fruchtbar gemacht wurde. Jakob Graf hält dem entgegen, 
dass diese Perspektive die Machtverhältnisse auf den Märkten unterschlage und nicht in den Blick bekommt, dass Marktmacht und hierarchische Märkte zur Kernstruktur kapitalistischen Wirtschaftens gehören und eine Form darstellen, wie sich dominante Akteure Werte, Profite und Natur aneignen.

In zwei weiteren Beiträgen wird die Debatte über die imperiale Lebensweise fortgeführt. Christa Wichterich diskutiert das Konzept aus einer intersektionalen Perspektive. Sie argumentiert, dass die imperiale Lebensweise wesentlich auf Sorgearbeit beruht, die sie sich sowohl innergesellschaftlich als auch international aneignet. Die Krise der sozialen Reproduktion, die dieser "Careextraktivismus « verursacht, hat sich infolge der Pandemie dramatisch zugespitzt. Davon zeugen sowohl die Überlastung der Pflegekräfte als auch die Sorgelücken, die sich aufgrund der Pandemie-bedingten Grenzschließungen vergrößerten.

Ulrich Brand und Markus Wissen antworten auf Stefanie Hürtgens Kritik in der PROKLA 1/2020 und 2/2021. Die imperiale Lebensweise, so argumentieren sie, kann und muss im Kontext der Klassenstruktur kapitalistischer Gesellschaften gesehen werden. Zudem ist es, wie von Hürtgen betont, wichtig, die widerständige Subjektivität der Lohnabhängigen in ihrer Bedeutung für ein sozial-ökologisches Transformationsprojekt zu berücksichtigen. Allerdings ist Widerständigkeit nicht per se emanzipativ, ökologische und soziale Interessen können trotz ihrer "Gleichursprünglichkeit« in konkreten Konflikten durchaus divergieren. Gegen Hürtgens Annahme »vergleichba- rer Raumfragmente « in Nord und Süd argumentieren Brand und Wissen, dass sich der Klassengegensatz aufgrund internationaler Abhängigkeitsverhältnisse räumlich sehr unterschiedlich ausprägt.

\section{$* * *$}

Wir feiern dieses Jahr nicht nur das 50-jährige Bestehen der PROKLA, sondern ein weiteres Jubiläum: Dorothea Schmidt ist seit 25 Jahren Mitglied der Redaktion. Als sie dazu stieß, so ist im Protokoll der Mitgliederversammlung von $1996 \mathrm{zu}$ lesen, bildeten erstmals nach 25 Jahren, also seit der Gründung der PROKLA, Frauen die Mehrheit in der Redaktion. Bereits 1993 war sie Gastredakteurin beim Schwerpunkt »Frauen in der Ökonomie (Heft 93) und steuerte einen Beitrag bei, der bereits andeutete, wo sie in den folgenden Jahren einen Schwerpunkt legen sollte: sozialwissenschaftliche Begrifflichkeiten sozialgeschichtlich gegen den Strich bürsten. Dorothea zeigt in ihren Texten immer wieder, dass in der kritischen Literatur vollkommen selbstverständlich verwendete Begriffe Ambivalenzen nicht zulassen oder gar die Vergangenheit verklären: der Fordismus stellte keinen neuen Typ von Kapitalismus dar, der sich derart auf den Begriff bringen lasse (Heft 172) und die arbeitende Klasse war auch früher alles andere als eine homogen Größe (Heft 175). Sie legt immer wieder den Finger in die Wunde, wenn die kritische Wissenschaft es dann doch nicht so genau wissen wollte. So weist sie in PROKLA 93 auf, dass die Technik, die Mechanisierung nicht zu einer Nivellierung, sondern im Gegenteil, zu einer Segmentierung bei der geschlechtsspezifischen Arbeitsteilung 
führte. Diese Entwicklung lag weniger im Interesse der Unternehmen, sondern war ein Resultat im Prozess der sozialen Konstruktion von geschlechtsspezifischem Arbeitsvermögen. Nicht das bornierte Interesse am Profit war treibende Kraft, sondern die Eigenlogik patriarchaler Verhältnisse, die sich überall einschrieb. Einen weiteren »Mythos « widerlegt sie etwa in Heft 117, wo sie die Tätigkeiten der Selbstständigen in Deutschland um 1900 und den 1990er-Jahren vergleicht. Dabei zeigt sie, dass die Vorstellung trügt, dass um die Jahrhundertwende die Erwerbstätigen mehrheitlich in industriellen Großbetrieben beschäftigt gewesen seien. Zwar sei die Zahl der Selbstständigen im 20. Jahrhundert lange Zeit rückläufig gewesen, doch war ihre Bedeutung um 1900 hoch. Damit nicht genug: Auch zum Ende des 20. Jahrhunderts hin war die Zahl der Selbstständigen wieder steigend. Dorothea konnte durch ihre genaue Analyse darauf hinweisen, dass die Wende zum 21.Jahrhundert, was die Bedeutung der Selbstständigen betrifft, »mehr strukturelle Ähnlichkeiten mit den Verhältnissen an der Wende zum 20. Jahrhundert auf[weist], als man zunächst denken würde" (Heft 117: 611). Vor allem historischen Themen bleibt Dorothea Schmidt treu, kein Wunder: 1996 wurde sie Professorin für Wirtschafts- und Sozialgeschichte an der HWR Berlin (vormals FHW), wo sie bis 2011 lehrte. Davor war sie über 16 Jahre in vor allem in Bremen Lehrbeauftragte und wissenschaftliche Mitarbeiterin und bereits Mitglied des Herausgebervereins der PROKLA. Seit zehn Jahren, also seit ihrer Pensionierung, hat sie noch mehr Zeit für die redaktionelle
Arbeit, wofür wir äußerst dankbar sind. In den letzten Jahren hat sich in und um die PROKLA allerhand bewegt, was viel zusätzliches Engagement bedurfte und immer wieder auch Dorotheas gesättigte Erfahrung, um Kurs zu halten. Über viele Jahre war sie Gastgeberin für die Redaktionssitzungen (noch nicht vom Tisch ist ein Rezeptbuch, mit den über all die Jahre gekochten Gerichten), bis sie, nach ihrer Pensionierung, wieder nach Wien zog. Das war auch ein Grund, warum wir bereits vor der Pandemie, Redaktionssitzungen mit Online-Unterstützung abgehalten haben. Dorothea ist nicht nur das Rückgrat der Redaktion und ist als Übersetzerin und Korrekturleserin unersetzlich. Als PROKLA-Autorin sind die Themen, derer sie sich annimmt, vielfältiger als die Ausführungen oben nahelegen: In jüngeren Texten wirft Dorothea etwa einen Blick auf die sozialen Aspekte der Russischen Revolution (Heft 200), oder analysiert - zusammen mit Friederike Maier - kritisch die aktuelle Care-Debatte (Heft 195). Besonders hervorzuheben ist jedoch ihr Engagement gegen Militarismus, Rüstung und Kleinwaffen (Hefte 127, 143, 162, 183, 201). Liebe Dorothea, hiermit wollen wir dir für deine so wichtige Arbeit in der Redaktion danken. Wir freuen uns auf viele weitere gemeinsame Jahre.

$$
* * *
$$

Mit der ersten Ausgabe in 2022 werden wir das Digital-Abo und das Print-Digital-Kombi-Abo einführen. Außerdem differenzieren wir die Preise aus. Für Institutionen, also all jene Abonnent*innen, die wir als Mehrfachleser*innen einstufen, erhöhen wir den Preis automatisch 
um 10 Euro. Diejenigen Abonnent*innen, die Ihr Abo umstellen oder in eine andere Preiskategorie wechseln möchten, bitten wir, dies über die Seite www. bertz-fischer.de/abo-wechsel zu tun. Wir freuen uns sehr, wenn diejenigen, die es sich leisten können, ein FörderAbo abschließen, denn wir müssen die ermäßigten Abos, die es ab 2022 auch erstmalig geben wird, gegenfinanzieren. Eine Preisübersicht finden Sie in dieser PROKLA auf Seite 590 und im Netz unter www.bertz-fischer.de/PROKLAabopreise.

$* * *$

Anfang August 2021 fand in Berlin die diesjährige Mitgliederversammlung der Vereinigung zur Kritik der politischen Ökonomie e.V. statt. In neuer Zusam- mensetzung wurde der Vorstand wiedergewählt: Nach vierzehnjähriger Vorstandstätigkeit wird Rudi Schmidt von Michael Heinrich als erster Vorsitzender abgelöst. Rudi Schmidt wurde für die vierzehnjährige Vorstandstätigkeit gedankt. Heinrich rief nochmals in Erinnerung, dass die letzten Jahre nicht leicht waren und sich Schmidt stark engagierte, die Gründung des Beirats vorschlug und mit Engagement dessen Arbeit begleitete. In fast bisheriger $\mathrm{Zu}-$ sammensetzung wurde der Redaktionsbeirat der PROKLA gewählt. Schmerzlich vermisst werden die beiden bisherigen Mitglieder Ulf Kadritzke und Wolfgang Schöller. Neu gewählt wurde in bisheriger Zusammensetzung auch die Redaktion.

\section{Der PROKLA-Förderverein}

Die PROKLA erscheint seit 1971 und bietet politisch engagierte sozialwissenschaftliche und ökonomische Analysen. Allein von den Verkaufserlösen kann sich die PROKLA nicht finanzieren, und in die Abhängigkeit von Parteien oder großen Verlagen wollte sie sich nie begeben. Deshalb wird die PROKLA von einem Förderverein herausgegeben, der "Vereinigung zur Kritik der politischen Ökonomie e.V.«, die jährlich in ihrer Vollversammlung die Redaktion der Zeitschrift wählt und die nächsten Themenschwerpunkte diskutiert.

Kritische Sozialwissenschaft kann nicht dem Markt überlassen werden. Ohne solidarische Strukturen und finanzielle Unterstützung sind Zeitschriften wie die PROKLA kaum möglich. Die finanziellen Beiträge der Vereinsmitglieder ermöglichen das Erscheinen der PROKLA, sie schaffen die Voraussetzungen für Kontinuität und Planbarkeit, wie sie für die Redaktionsarbeit unabdingbar sind. Wir freuen uns über weitere Mitglieder, regelmäßige Spenden oder einmalige Zuwendungen. Weitere Informationen teilen wir gerne per E-Mail mit (redaktion@prokla.de).

Vereinigung zur Kritik der politischen Ökonomie e.V. | Postbank Berlin | IBAN: DE17 100100100538135100 | BIC: PBNKDEFF 